\title{
GOFRSETI
}

\section{¿Qué queremos regular? Algunos apuntes para la regulación de la industria Fintech}

\author{
José Villafuerte Mendoza*
}

Resumen. - En la actualidad, la industria Fintech dentro del mercado financiero está revolucionándolo a través de desarrollos tecnológicos para poder beneficiar a las empresas en diferentes rubros dentro de esta. Sin embargo, debido a la reciente entrada de la tecnología Fintech, se proponen algunas recomendaciones para poder regular esta industria en el Perú, y así no se perjudique su desarrollo a largo plazo.

\begin{abstract}
At present, the industry Fintech inside the financial market is revolutionizing it across technological developments to be able to be of benefit to the companies in different items inside this one. Nevertheless, due to the recent entry of the technology Fintech, it is proposed some recommendations to be able to regulate this industry in Peru, and this way his long-term development is not harmed.
\end{abstract}


Cuando uno piensa en la industria Fintech tiene la tendencia a imaginar empresas que desarrollan nuevas plataformas para proveer los mismos productos que hoy ofrece la banca tradicional. Pero esa es una forma muy limitada de entender a las Fintech. Estas empresas no solamente desarrollan nuevas plataformas, sino que están cambiando radicalmente el producto financiero mediante desarrollos tecnológicos enfocados en eliminar las ineficiencias de los productos financieros y, como consecuencia de ello, incrementar los potenciales márgenes de rentabilidad reduciendo costos de transacción, riesgos operacionales y de crédito.

Pensemos, por ejemplo, en una aplicación que realiza una calificación crediticia basada en información no tradicional, como el comportamiento del consumidor en redes sociales. En este tipo de aplicación, actualmente utilizado por varias empresas Fintech en el Perú, las publicaciones que usted haya efectuado en plataformas como Facebook, Twitter, LinkedIn o Instagram, podrían modificar su calificación crediticia porque la aplicación evaluaría, por ejemplo, cuántos viajes realiza usted al año, cuál es su lugar de trabajo, cuántas denuncias tienen sus publicaciones y cuantos usuarios lo han bloqueado.

Otro ejemplo de una tecnología disruptiva en el mundo Fintech lo tenemos en plataformas de asesoría de inversiones, con algoritmos que permiten invertir en posiciones o retirarse de ellas con mayor rapidez y sin depender de la capacidad de reacción de las personas encargadas de un portafolio de inversiones. De la misma forma, dentro del mundo de las Fintech también encontramos a la criptomonedas o monedas virtuales, que, mediante su emisión digital, descentralizada y sin intervención del gobierno, está revolucionando el mundo y creando dolores de cabeza a los bancos centrales.

Como vemos, la tecnología Fintech no es una adaptación digital de los típicos productos de la banca tradicional, sino que son esencialmente tecnologías disruptivas que están revolucionando el mercado financiero por las siguientes razones ${ }^{1}$ :

(i) El desarrollo de la tecnología es exponencial en todos los ámbitos, y la tecnología Fintech no es una excepción. La capacidad de los procesadores, la complejidad de los algoritmos y la velocidad de conexión a internet tienen un crecimiento acelerado que se refleja inmediatamente en sus aplicaciones. Este crecimiento exponencial no será posible en la banca tradicional, acostumbrada a un desarrollo más pausado y más dependiente de la decisión humana.

(ii) La tecnología Fintech tiende a eliminar la necesidad de los intermediarios tradicionales en el mercado financiero, por ejemplo, plataformas de préstamos peer-to-peer, que originalmente requerían de la intermediación de una empresa del sistema financiero. Las plataformas Fintech usualmente conectan

1 BRUMMER, Chris y GORFINE, Daniel. “Fintech: Building a 21st century regulator's toolkit”. Milken Institute Center for Financial Markets, 2014. 
de una forma directa a agentes deficitarios con agentes superavitarios de capital.

(iii) El desarrollo de las Fintech genera que la banca tradicional y las empresas de tecnología, vinculadas directamente o no al mercado Fintech, compitan por los mismos consumidores o por algunos segmentos del mercado.

(iv) Comparado con la banca tradicional, el mercado Fintech tiene muy pocas barreras de acceso y bajos costos asociados, al prescindir, en la mayoría de los casos, de presencia física y de equipos masivos de atención al cliente.

(v) El Internet permite que el mercado Fintech se desarrolle sin fronteras, creando un solo mercado global en el que una compañía puede llegar a consumidores en cualquier parte del mundo.

(vi) El mercado Fintech ha permitido la democratización de las oportunidades de inversión, ha reducido los costos transacción en el mercado financiero y ha incrementado la inclusión financiera.

Estas características nos permiten ver que el fenómeno Fintech está muy lejos de ser comprendido en su totalidad. Aún es muy pronto para poder tener certeza de todos los efectos que tendrá esta revolución dentro del mercado financiero y dentro de la economía global. Sin embargo, es entendible que todos los reguladores, y en general los gobiernos de la mayoría de países en el mundo, hayan reaccionado con preocupación y hayan activado sus alertas internas sobre el desarrollo de estas tecnologías.

En esa línea, hemos notado con cierta preocupación la presentación de un proyecto de ley en el Congreso de la República que propone declarar de interés nacional y necesidad pública la regulación de la tecnología financiera Fintech "con el objetivo de proteger los intereses de los usuarios".

Nuestra inquietud evidentemente no está sustentada en que creamos que los intereses de los usuarios o consumidores no deban protegerse, sino por el contrario, porque creemos que esta ley podría generar decisiones apresuradas en nuestros organismos reguladores que finalmente puedan ser contraproducentes, afectando a quienes intentábamos proteger.

Por supuesto, la tecnología Fintech trae consigo nuevas preocupaciones, como la dificultad de conocer exactamente todos los riesgos operacionales a los que se encuentra sujeta, o la imposibilidad de crear restricciones absolutas de actividades que finalmente pueden proveerse desde cualquier otra parte del mundo a través de internet. Sin embargo, la tecnología Fintech también revive preocupaciones ya vividas con la banca tradicional nos referimos por ejemplo al riesgo sistémico que, según algunos autores, estas nuevas tecnologías replican con distintas características. 
Magnuson², por ejemplo, considera que, a pesar del menor tamaño de las Fintech, el riesgo sistémico que estas compañías traen consigo es comparable con el riesgo sistémico que representan las entidades de la banca tradicional conocidas como compañías "too big to fail"; es decir, aquellas que, por su tamaño e importancia en el mercado, y por su conexión con otros actores de la economía, pueden ocasionar un desastre económico si llegaran a caer.

Aunque, desde nuestra perspectiva, y reconociendo que las compañías Fintech son aún mucho más pequeñas que la banca tradicional, es exagerado comparar el riesgo sistémico de las compañías que generaron la crisis económica del 2008 con el riesgo sistémico generado por las compañías Fintech, que tienen una presencia menor en el mercado, un tamaño menos considerable de inversiones y una administración más reducida de activos de terceros; es cierto, como apunta dicho autor, que las compañías Fintech tienen menor aversión al riesgo en sus decisiones de inversión y por lo tanto sus políticas de reducción de riesgo operacional, crediticio y sistémico son más disipadas que las aplicadas por la banca tradicional; sin embargo, consideramos que esta menor aversión al riesgo no es una característica propia de las compañías Fintech, sino que es una característica que viene ligada con el concepto propio de un emprendimiento.

Para explicar nuestro punto, quisiéramos que el lector se ponga en el supuesto de cualquier emprendimiento quiera efectuar. La decisión propia de desarrollarlo implica asumir muchos más riesgos, en un momento determinado, que los riesgos asumidos por cualquier compañía con años de experiencia en el mercado, por desarrollar un nuevo producto. La idea central del emprendimiento es ser disruptivo y conseguir un tamaño tal que nos permita retornos en el más breve plazo para garantizar su sostenibilidad en el tiempo.

En ese sentido, nos parece relevante, como explicaremos en los siguientes párrafos, que centremos bien el tema de los riesgos asumidos por una compañía del sector Fintech. Estos riesgos se asumen, en principio, por su naturaleza de emprendimientos (por lo menos en esta etapa de su desarrollo) y no necesariamente porque sea una característica propia de las compañías Fintech.

Si bien es cierto el riesgo sistémico se incrementa en situaciones donde los actores son más vulnerables a los embates de la economía, y un actor es más vulnerable en la medida que su tamaño sea más reducido, esto por si sólo no convierte a las Fintech en actores cuyo riesgo sistémico sea necesariamente comparable con la banca tradicional, sobre todo porque para la existencia de riesgo sistémico se requiere también que existan mecanismos de propagación de su caída a otros sectores de la economía, algo que dependerá del tamaño y complejidad que pueda alcanzar actor. Es bastante discutible que, en esta etapa del desarrollo de las

2 MAGNUSON, William J.“Regulating Fintech”, Vanderbilt Law Review, Texas A\&M University School of Law Legal Studies, Research Paper 17-55, Agosto 26, 2017. Disponible en SSRN: https:/ / ssrn.com/abstract=3027525. 
Fintech, que tienen aún muchas características de un emprendimiento, se haya alcanzado un tamaño y complejidad tal que la caída de una de ellas genere un efecto dominó en todo el sistema y en otros sectores de la economía.

Se ha dicho también que las Fintech generan mayor riesgo sistémico porque la asimetría de información en dicha industria es muy elevada. Sin embargo, creemos que esta característica no revela necesariamente que las Fintech generen un riesgo sistémico considerable, sino que, por el contrario, revelan que conocemos tan poco de la industria que tomar decisión de regulación basado en tan poca información carece de sentido y podría ser contraproducente para la economía.

Dejando de lado el espinoso tema del riesgo sistémico, creemos que la regulación de la industria Fintech merece un análisis más profundo que el simple deseo, bien intencionado, de proteger a los consumidores. Es indiscutible la necesidad de desarrollar una industria Fintech en el Perú, que promueva la inclusión financiera, el desarrollo de nuevos emprendimientos, la creación de riqueza y mayor competencia en el mercado financiero que se traduzca en mejores condiciones para los consumidores. Sin embargo, para que eso suceda, se debe tener especial cuidado en el marco regulatorio que se desee aplicar a esta industria, pues, como sabemos, existen regulaciones en otros sectores que tuvieron muy buenas intenciones pero que acabaron con la industria.

En ese sentido, nos permitimos mencionar algunos puntos a tener en consideración en el estudio de las Fintech para su posterior regulación, con la finalidad de evitar, en lo posible, dañar una industria que apenas se encuentra naciendo en el Perú:

(i) Sin industria no habrá regulación: Si partimos de la premisa que nuestro objetivo es permitir el desarrollo de una industria Fintech responsable y que tenga en cuenta los derechos de los consumidores; el paso previo es permitir que esta industria exista. En esa medida, se debe considerar, como mencionamos en párrafos anteriores, que la industria Fintech se desarrolla sin ningún tipo de fronteras, y que, eventualmente, cualquier compañía del mundo puede proveer servicios financieros en el Perú a través del internet. No es extraño, por ejemplo, recibir préstamos menores o asesoría financiera a través de aplicaciones móviles o plataformas tecnológicas administradas por compañías asentadas en otras partes del globo.

En consecuencia, el regulador deberá tener en consideración que las normas que establezca para su aplicación a compañías en el Perú, tienen que permitirles competir en el mercado, esto implica que no deberán crearse sobrecostos regulatorios excesivos que generen barreras de entrada a las compañías Fintech o que impliquen un considerable incremento de los costos de transacción que hagan imposible que estas compañías disputen el mercado con sus pares alrededor del mundo. 
(ii) Dejemos que la competencia comience: La competencia en el mercado financiero, como en cualquier otro mercado, ha demostrado ser beneficiosa para los consumidores, quienes obtienen productos y servicios en mejores condiciones y a menor costo. En ese sentido, si el objetivo del regulador es promover la competencia y la inclusión financiera en el Perú, resulta primordial que las Fintech participen en esta competencia y mitiguen la concentración en el mercado financiero.

En consecuencia, si se pretende regular a las Fintech, entonces también es necesario que las dotemos de condiciones y beneficios similares a las empresas financieras tradicionales, de lo contrario, estaríamos condenando a la industria a ser menos eficientes que las empresas tradicionales por mandato legal y no por su comportamiento en el mercado o su capacidad de innovación.

Por ejemplo, podemos notar que, bajo la regulación peruana, únicamente las empresas tradicionales del sector financiero, actualmente reguladas, tienen la posibilidad de fijar libremente las tasas de interés y comisiones que aplican en sus operaciones. Sin profundizar demasiado en este tema, que puede ser materia de un estudio mucho más amplio, debemos precisar que existen diversos estudios que han demostrado que la limitación de tasas de intereses no es efectiva para la reducción de los costos de financiamiento, y que, por el contrario, generan la marginación de un segmento de potenciales consumidores, reducen la transparencia en el mercado y reducen la competencia 34 .

En ese sentido, resulta razonable que, agregando costos regulatorios a la industria Fintech, también sea necesario dotarles de estos beneficios y libertades para que puedan competir en el mercado financiero. De lo contrario, estaríamos creando una regulación completamente diferenciada en perjuicio de una industria nueva e, indirectamente, estaríamos reduciendo la competencia en el mercado en perjuicio de los consumidores.

(iii) No todas las Fintech son iguales: Otro error a evitar en el marco regulatorio es asumir que las empresas del sector Fintech desarrollan actividades similares o comparables y que, en esa medida, puedan compartir un set regulatorio similar o general. Nada más lejano de la realidad.

Como hemos visto en párrafos precedentes, la industria Fintech se viene desarrollando en distintos sectores con diferentes propósitos e ideas de negocio y con características disímiles. No son comparables, por ejemplo, una compañía que se dedique a otorgar préstamos con sus propios recursos a favor de terceros mediante una aplicación móvil, con una compañía que haya desarrollado una criptomoneda. Estamos hablando de riesgos totalmente distintos, con diferentes

3 STATEN, Michael. "The Impact of Credit Price and Term Regulations on Credit Supply" Joint Center for Housing Studies Harvard University, 2008.

4 VANDENBRINK, Donna C. "The effects of usury ceilings". Economic Perspectives, 1982. 
niveles de complejidad y cuya interacción con los demás sectores de la economía será también particular.

Dicho esto, consideramos que la regulación, de existir, tiene que centrarse en actividades y no compañías y, mucho menos, industrias heterogéneas. Existen actividades, desarrolladas por compañías Fintech, que podrían merecer regulación particular y aquellas otras que no requieren de mayor intervención estatal. Asimismo, existen actividades que requieren solamente regulación local y aquellas otras, híper descentralizadas y dispersas en el globo, que requieren una mayor coordinación de esfuerzos entre reguladores de distintos países. Finalmente, existen actividades cuyo tamaño e interconexión genera un riesgo sistémico importante, y aquellas otras que no representan mayor preocupación al respecto. La industria Fintech es heterogénea y su regulación no podría ser distinta. No puedes regular aquello que no entiendes: La industria Fintech trae consigo nuevos retos para todos los que, de alguna u otra forma, estamos vinculados al mercado financiero, y los organismos reguladores no son la excepción.

La alta complejidad tecnológica de esta industria, sumada con el poco conocimiento que, sobre la materia, poseen generaciones con menor alfabetización digital, es una ecuación complicada para cualquier especialista en temas financieros. Algoritmos kilométricos, programas completamente autónomos, inteligencia artificial, manejo de información masiva en tiempo récord, son algunos ejemplos de las cosas con las que hoy tenemos que lidiar y que entendemos muy poco.

En ese sentido, es clave que el regulador no solamente esté familiarizado con estos términos, sino que conozca su significado y su proceso a profundidad. De lo contrario, el riesgo de emitir disposiciones legales que acaben con el sector es alto. Imaginemos por ejemplo una norma que, so pretexto de la seguridad jurídica, pretenda obligar a que los contratos digitales celebrados con empresas Fintech se realicen utilizando el mecanismo de firmas digitales (un proceso bastante más complejo y costoso que un simple clic en un enlace de aceptación). Estaríamos condenando a las empresas Fintech a elevar sus costos de transacción y a celebrar operaciones únicamente con consumidores sofisticados y con conocimientos sobre contratación digital.

Somos conscientes de que la curva de aprendizaje es alta, y que nos tomará bastante tiempo comprender plenamente las tecnologías y la lógica económica que está detrás de la industria Fintech, pero creemos, sin temor a equivocarnos, que el aprendizaje sobre el sector es un requisito indispensable para una regulación responsable y que cumpla su objetivo sin dañar a la industria.

(iv) La innovación no es el enemigo: Existe una tendencia bastante humana de preocuparse por cambios que alteran el statu quo de las cosas. Esta preocupación es aún mayor cuando estos cambios son dramáticos y se 
producen con velocidades que no podemos rastrear con nuestros sentidos o percibir de una manera intuitiva.

El cambio tecnológico es así de dramático, es difícil seguir su paso y cada nuevo programa, aplicación o procesador es exponencialmente superior a la versión previa. En ese sentido, la innovación tecnológica es un factor a considerar en la industria Fintech cuyo negocio principal se desarrolla utilizando dichas innovaciones.

Por lo tanto, desde nuestra perspectiva, la labor del regulador será promover disposiciones legales lo suficientemente flexibles para permitir la innovación y adaptarse a ella. No parece una labor fácil, pero el conocimiento profundo del sector puede dotarnos de las herramientas necesarias para conseguir esta flexibilidad. Por ejemplo, es evidente que disposiciones rígidas sobre riesgo operacional podrían ser un obstáculo para la innovación tecnológica puesto que la innovación presupone cambios constantes en los sistemas que modifique, por ejemplo, los estándares de seguridad de la información o de los procesos internos de una compañía.

(v) El mandato constitucional es proteger el ahorro: Como sabemos, el artículo 87 de nuestra Constitución Política, establece que es labor del estado el fomento y protección del ahorro. Esta piedra angular, resulta siendo el soporte constitucional de toda la regulación en el mercado financiero.

En ese sentido, a pesar de que, como sabemos, los objetivos de dicha regulación han evolucionado en el tiempo, y, en particular, sobre la base de riesgos adicionales detectados en el mercado financiero a nivel mundial, siempre será una buena idea reformular nuestros conceptos y prioridades cuando pretendamos regular un aspecto de la economía.

En consecuencia, consideramos recomendable que el regulador tenga en consideración que el objetivo fundamental de su participación en el mercado es proteger el ahorro, y, donde no haya ahorro o captación de recursos del público, tendrá que evaluarse con mayor detenimiento si es necesario o no regular y cuáles son los aspectos, distintos al ahorro, que queremos proteger o evitar. Este ejercicio es fundamental para evitar los sobrecostos regulatorios que puedan poner en desventaja a las empresas reguladas frente a sus pares del exterior o frente a proveedores distintos del mismo servicio no sujetos a regulación alguna. En ese sentido, las inquietudes de los organismos reguladores son razonables siempre y cuando exista mandato constitucional para proteger algún aspecto en particular de la economía o fundamentos claros y concretos de la existencia de algún riesgo adicional que quiera mitigarse. Desde nuestro punto de vista, este es otro de los pilares fundamentales de una regulación responsable.

(vi) Debemos tener claro el riesgo que queremos mitigar: En línea con lo comentado en el punto anterior, el ejercicio de la labor regulatoria deberá centrarse primero en conocer los riesgos que afrontan las compañías de la 
industria Fintech. Tal como comentamos en puntos anteriores, existen, por ejemplo, autores que argumentan la posible existencia de un riesgo sistémico importante en el sector. Sin embargo, toda esta literatura debe ser confrontada con la realidad de la industria en el país. Es por lo menos cuestionable, por ejemplo, que actualmente pretendamos hablar de algún riesgo sistémico generado por la industria Fintech en el Perú, considerando el tamaño de sus operaciones y el incipiente desarrollo de los emprendimientos en comparación con el mismo sector en otras jurisdicciones. La labor del regulador, entonces, será medir los riesgos adecuadamente y evaluar la necesidad de imponer alguna regulación si es que los riesgos efectivamente existen y son considerables.

Finalmente, y a manera de conclusión, consideramos atendible la propuesta de que la mejor respuesta regulatoria a la industria Fintech pasa por la creación de un área de pruebas (sandbox) regulatoria. Este sandbox, tal como ha sido pensado y creado en otros países, por ejemplo, el Reino Unido y México (que resulta ser el país con mayor desarrollo de la industria Fintech de Latinoamérica), permitiría al regulador acompañar al desarrollo de esta industria, con la finalidad de entender con profundidad sus procesos y los riesgos asociados, y diseñar, en paralelo, una regulación particular y adaptada a la industria.

$\mathrm{Al}$ respecto, tal como ha indicado el propio regulador británico (Financial Conduct Authority - FCA) en un informe publicado en noviembre de 2015, el propósito de este sandbox regulatorio es conseguir un mayor acceso al mercado financiero, incrementar la competencia en el sector y reducir los costos de transacción, todo en beneficio de los consumidores. Este sandbox regulatorio británico (denominado Project Innovate) permitiría a las compañías iniciar operaciones con autorizaciones restringidas para realizar pruebas de los modelos de negocio hasta que tengan el tamaño adecuado para cumplir con todos los requisitos de la regulación. Asimismo, el regulador efectúa un seguimiento y guía individual a las compañías para conocer sus procesos y adaptar las regulaciones a cada segmento del sector y renuncia temporalmente al inicio de procedimientos sancionadores mientras dure el periodo de prueba de los modelos de negocio.

Como vemos, y regresando a la preocupación que manifestamos al inicio de este artículo sobre el interés por la regulación inmediata de la industria, creemos recomendable explorar primero respuestas intermedias y previas al inicio y creación de regulaciones en el sector Fintech (como, por ejemplo, la creación de un sandbox regulatorio), con la finalidad de evitar una regulación apresurada y contraproducente, que desincentive la inversión en el sector y que, como consecuencia de ello, perjudique la competencia y a los consumidores. 